\title{
Robotic-assisted Ivor Lewis esophagectomy with combined stapled/sewn anastomosis
}

\author{
Deven C. Patel, Evangeline Rodriguez, Fernando Espinoza-Mercardo, Taryne A. Imai, Harmik J. Soukiasian \\ Division of Thoracic Surgery, Department of Surgery, Cedars-Sinai Medical Center, Los Angeles, CA, USA \\ Contributions: (I) Conception and design: DC Patel, HJ Soukiasian; (II) Administrative support: DC Patel, HJ Soukiasian; (III) Provision of study \\ materials or patients: DC Patel, HJ Soukiasian; (IV) Collection and assembly of data: DC Patel, TA Imai, HJ Soukiasian; (V) Data analysis and \\ interpretation: All authors; (VI) Manuscript writing: All authors; (VII) Final approval of manuscript: All authors. \\ Correspondence to: Harmik J. Soukiasian, MD. Professor of Surgery, Chief, Division of Thoracic Surgery, Vice-Chairman, Department of Surgery, \\ Cedars-Sinai Health System, 8631 W. 3rd St., Suite \#240 East, Los Angeles, CA 90048, USA. Email: Harmik.Soukiasian@cshs.org.
}

\begin{abstract}
The esophagectomy remains the primary modality of treatment for localized esophageal cancer. A formidable operation for surgeons given its technical complexity and associated morbidity and mortality. Accordingly, a minimally invasive approach has become increasingly utilized given the reduced perioperative morbidity, shortened hospital stay, and improved patient satisfaction when compared to the traditional open esophagectomy. A variety of surgical techniques exist for the construction of the esophagogastric anastomosis, including a hand-sewn, circular stapled, linear stapled, or a combined stapled/sewn (modified Collard) approach. In this article, we detail our two staged approach to the robotic-assisted Ivor Lewis esophagectomy with combined stapled/sewn anastomosis.
\end{abstract}

Keywords: Robotic Ivor Lewis esophagectomy (RILE); minimally invasive surgery; esophageal cancer; combined stapled/sewn anastomosis; modified Collard

Received: 16 November 2019; Accepted: 31 January 2020; Published: 05 October 2020.

doi: 10.21037 /jovs.2020.02.08

View this article at: http://dx.doi.org/10.21037/jovs.2020.02.08

\section{Introduction}

Over a century ago, Franz A. Torek performed the first transthoracic esophagectomy (1). Since then the procedure has greatly evolved with improved outcomes and a wide variety of techniques. The most recent trend has been the minimally invasive esophagectomy (MIE). This approach has garnered a significant amount of interest given the documented reduction in perioperative morbidity, faster recovery from surgery, and improved quality of life for patients without compromising oncologic principles (2).

The newest advancement to the MIE is the robotic platform. This innovative technology offers benefits not available with traditional laparoscopic and thoracoscopic methods. Including high resolution three-dimensional optics, providing visual-spatial relationships of anatomical structures during dissection. Other benefits include, improved range of motion with wristed instruments, as well as stapler technology using real time tissue thickness feedback to optimize the integrity of staple lines. Additionally, the robotic platform has started to change the paradigm of surgical training and practice, enabling surgeons with little minimally invasive surgery background to quickly adopt the technology (3). Although the use of the robot has not yet demonstrated any survival benefit or clear advantages over the traditional MIE, there have been promising outcomes from specialized centers who have refined their technique through experience and process improvement $(4,5)$.

A variety of techniques have been described for the esophagogastric anastomosis, most commonly utilized are the hand-sewn, circular stapled, linear stapled, and the modified Collard (combined stapled/sewn) technique. There is no general consensus on the ideal anastomosis, however, the primary goals include avoiding postoperative leaks, dysphagia, and stricture formation (6). Collard et al. 
first described the combined stapled/sewn anastomosis, as a terminalized semi-mechanical side-to-side suture technique of a cervical esophagogastrostomy (7). Effectively, the posterior walls of the esophagus and stomach are placed in apposition so a linear cutting stapler can be applied to construct the posterior wall of the anastomosis, then the anterior wall is constructed using a running suture. The anterior wall is closed in a transverse fashion, which enables maximal distraction of the stapled ends of the posterior wall and increases the cross-sectional area of the anastomosis (8).

We will outline our surgical technique and considerations for a robotic Ivor Lewis esophagectomy (RILE) using a combined stapled/sewn anastomosis. Additionally, as the value of an experienced team has proven to favorably influence outcomes for patients undergoing an esophagectomy, the roles of the multidisciplinary surgical team will be highlighted (9).

\section{Preoperative considerations and multidisciplinary team}

Given the complexity of esophageal cancer, a thorough preoperative evaluation using a multidisciplinary approach is essential. Additional considerations include obtaining a thorough surgical and medical history, as well as an evaluation of lung function (as per standard pulmonary function testing). Once a patient is determined to be a surgical candidate, surgical approach is typically dependent on the location of the esophageal carcinoma. The RILE is often employed for middle to distal esophageal lesions and permits direct visualization of the thoracic esophagus, enabling a complete thoracic lymphadenectomy. Additionally, an intrathoracic esophageal anastomosis has been associated with better functional and physiologic results; with less dysphagia, less benign anastomotic strictures requiring fewer dilations, and a lower incidence of recurrent laryngeal nerve palsy when compared to a cervical anastomosis (10).

Given the intricacies of the operation, performing an esophagectomy requires a well concerted team effort. Often the anesthesiology team is experienced in thoracic cases, and understand the nuances of the RILE. They are in constant communication with the surgical team. The surgical technologists participating in the case undergo formal training of the robotic da Vinci ${ }^{\circledR}$ system (Intuitive Surgical Inc., Sunnyvale CA, USA) and are experienced with the logistics of the device. The immediate surgical team consists of a senior general surgery resident or cardiothoracic surgery fellow who actively participate in the operation using a secondary da Vinci ${ }^{\circledR}$ robotic console that is adjacent to the primary console used by the attending surgeon of the case. Additionally, the team includes an experienced thoracic surgery physician assistant who remains scrubbed in at all times and utilizes the assistant port for both abdominal and thoracic components of the operation.

\section{RILE}

It is our practice to perform the RILE as a staged process. The first stage consists of a flexible bronchoscopy, esophagogastroduodenoscopy (EGD), robotic assisted staging laparoscopy, and vascular conditioning of the gastric conduit. If there is no evidence of metastatic disease or any abnormalities that would preclude the patient from proceeding, the patient returns to the OR the following day for the second stage and completion RILE. Of note, in our practice we do not routinely place feeding jejunostomy tubes. Instead we place them selectively based on a patient's nutritional status and ability to tolerate an oral diet postoperatively.

\section{Stage 1: diagnostic assessment and vascular conditioning of the gastric conduit}

The patient is placed under general anesthesia and endotracheal intubation using a single lumen tube, and positioned supine with both arms tucked. A flexible bronchoscopy is performed, assessing for possible airway involvement of the esophageal carcinoma and clearing out any secretions that may facilitate postoperative pulmonary complications. Next, an EGD is performed to confirm tumor location and rule out further progression of disease.

A Veress needle is placed at Palmer's point and the abdomen is insufflated to a pressure of $15 \mathrm{mmHg}$. Using an optical entry technique, an $8 \mathrm{~mm}$ robotic port is placed approximately $1-2 \mathrm{~cm}$ cephalad and $1 \mathrm{~cm}$ lateral to the umbilicus on the patient's left (will serve as the camera port, or robotic Arm 2). A $12 \mathrm{~mm}$ robotic port is then placed in the right lower quadrant (Arm 1), and an additional two $8 \mathrm{~mm}$ robotic ports are placed in the same plane as the camera port on the patient's left-approximately 8-10 cm apart (Arms $3 \& 4$ ) depending on the patient's abdominal width. A $12 \mathrm{~mm}$ assistant port is placed in the inferior RLQ, triangulated between Arm 1 port and the camera port. Maintaining approximately $8-10 \mathrm{~cm}$ spacing between all robotic ports prevents the arms from colliding. 


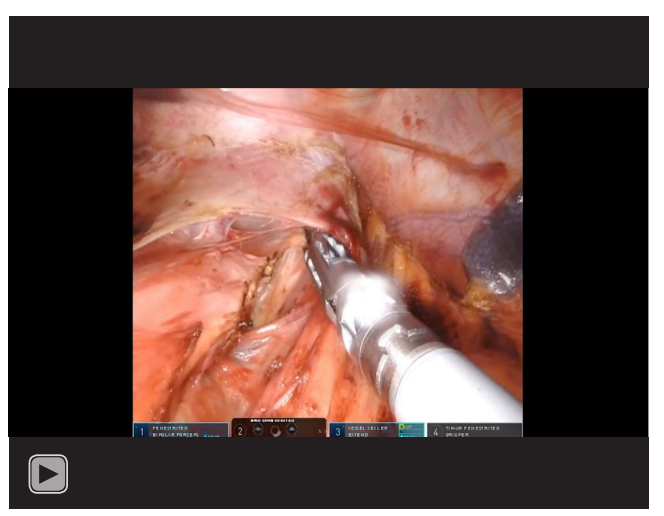

Video 1 Robotic Ivor Lewis esophagectomy (RILE) abdominal 1 trim.

Occasionally, the longer bariatric robotic ports can be used to assist in staggering the arms to optimize the range of motion. Furthermore, a Nathanson retractor is used to lift the left lobe of the liver and expose the diaphragmatic hiatus. Once all the ports are positioned, the patient is placed in a steep reverse Trendelenburg position, and the robotic system is docked. For the abdominal portion of the surgery, our da Vinci ${ }^{\circledR}$ instrument placement consists of: Arm 1: force bipolar/stapler, Arm 2: camera, Arm 3: vessel sealer, Arm 4: tip-up fenestrated grasper.

Next, the gastrohepatic ligament is divided using the robotic vessel sealer until the right diaphragmatic crus is visualized (see Video 1). It is important to maintain caution for a replaced left hepatic artery, encountered in approximately $4-10 \%$ of the general population (11). Next, the anterior border of the phrenoesophageal ligament adjacent to the right crus is incised, and the dissection is carried circumferentially-freeing the esophagus away from the left crus, and also clearing the retroesophageal space. The dissection is carried into the lower mediastinum in order to fully mobilize the distal portion of the esophagus. The visibility offered by the robotic camera enables an extensive dissection.

Once the lower esophagus is mobilized the gastrocolic ligament is divided. Throughout this dissection, visualization of the right gastroepiploic artery (RGA) is maintained at all times to avoid an inadvertent injury. Occasionally, indocyanine green (ICG) fluorescence is utilized for real-time visualization of the RGA using the Firefly ${ }^{\circledR}$ technology integrated into the da Vinci ${ }^{\circledR} \mathrm{Xi}$ system. During the dissection, view modes (from color to infrared) are switched through the console to ensure a safe distance from the RGA. Additionally, an omental flap along the greater curvature is maintained for later use as a buttress for the esophagogastric anastomosis. Division along the greater curvature is carried laterally and the short gastric arteries are transected using the robotic vessel sealer to the level of the left crus. The gastric dissection is continued inferiorly towards the pylorus, again staying a safe distance away from the origin of the RGA. Next, the stomach is retracted superiorly (by grasping the lesser curvature as to not traumatize the future gastric conduit). The retrogastric attachments are then transected and the celiac axis is identified. The left gastric artery and vein are divided using the robotic stapler (white load). At this juncture, a meticulous lymph node dissection is carried out ensuring removal of the nodes along the celiac and left gastric arteries.

This completes the first stage of the operation. All ports are removed and skin staples are placed at all of the port sites for ease of removal for the second stage of the operation the following day. The patient is normally permitted a liquid diet, and then made nil per os (NPO) after midnight.

\section{Stage 2: completion of Ivor Lewis esophagectomy}

\section{Abdominal portion}

The following day the patient is brought back to the OR and placed in the supine position under general anesthesia and endotracheal intubation. A double lumen endotracheal tube is placed in preparation for lung isolation during the thoracic component of the operation. All robotic ports, assistant port, and Nathanson retractor are placed identical to the previous day using the same incision sites with the exception of replacing Arm 1 with the robotic stapler. Once the robot is docked, the stomach is carefully examined, viability and perfusion are visually ensured. Next, the gastric conduit is formed by tubularizing the stomach using the robotic stapler (Video 2). The stomach is held superiorly to maintain organo-axial tension, and the first staple is fired at the incisura. Serial staple (black) loads are fired towards the gastric fundus away from the angle of his until the gastric conduit is completely divided free. Aiming to fashion a gastric conduit with a uniform $2-3 \mathrm{~cm}$ diameter. One of the notable features of the da Vinci ${ }^{\circledR}$ system is the SmartFire ${ }^{\mathrm{TM}}$ technology, ensuring a consistent staple line while minimizing tissue damage by obtaining real-time 


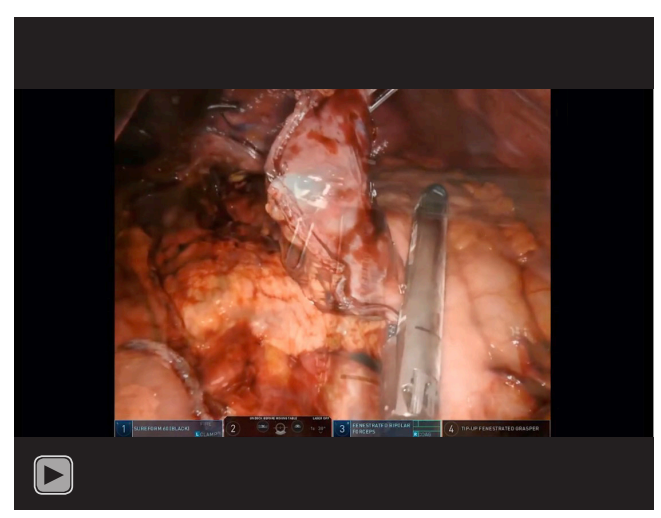

Video 2 Robotic Ivor Lewis esophagectomy (RILE) abdominal 2 trim.

measurements of the tissue thickness.

Moreover, the gastric conduit is then sutured to the future gastroesophageal specimen using a barbed, nonabsorbable 0 V-Loc ${ }^{\mathrm{TM}}$ (Medtronic, Minneapolis, MN, USA) suture. The suture bites include a $19 \mathrm{Fr}$ Blake drain that is inserted into the abdomen (Video 2). In theory, the drain acts as a buttress and dissipates the force used to later pull the specimen into the thorax. The robotic instruments are then removed, and all incisions are closed with staples. The 19 Fr Blake drain is securely sutured to the abdominal wall and will ultimately serve to drain the abdomen as well as the length of the conduit in the thorax. The robot is then undocked while maintaining sterility of the instruments so that the patient can be repositioned. Note, a gastric drainage procedure in the form of a pyloroplasty or pyloromyotomy is not performed, as there has not been clear evidence of its utility (12).

\section{Thoracic portion}

The patient is placed in the left lateral decubitus position with a slight anterior tilt and flexion of the OR table enabling optimal spacing of the rib spaces. The patient is placed on single lung ventilation by the anesthesiologist. The port placement is as follows: one $12 \mathrm{~mm}$ robotic port is placed in the posterior $8^{\text {th }}$ intercostal space (Arm 1), another $12 \mathrm{~mm}$ robotic port in the inframammary region/ midclavicular (Arm 4). A robotic $8 \mathrm{~mm}$ port is placed in the posterior axillary line, $9^{\text {th }}$ intercostal space (Arm 2), another $8 \mathrm{~mm}$ robotic port is placed in the $3^{\text {rd }}$ intercostal space anterior to the trapezius muscle (Arm 3). Finally, a $15 \mathrm{~mm}$ assistant port is placed in the posterolateral chest at the level of the diaphragm, triangulated between Arm 1 and

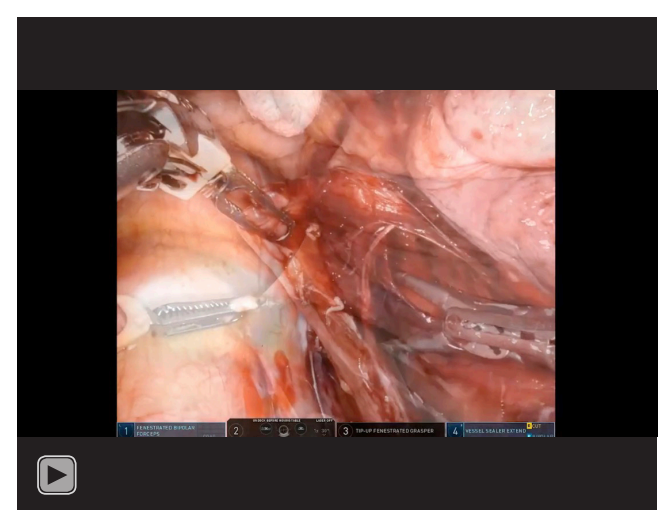

Video 3 Robotic Ivor Lewis esophagectomy (RILE) thoracic trim.

the robotic camera (Arm 2). The robot is then again docked, with instruments placed in the following manner: Arm 1: force bipolar, Arm 2: camera, Arm 3: tip-up fenestrated, Arm 4: vessel sealer.

As seen in Video 3, the lung is retracted anteriorly and the inferior pulmonary ligament is divided using the vessel sealer (lymph nodes encountered are handed off as a specimen). Next, the pleural reflection posterior to the esophagus is incised along the pericardium towards the subcarinal space, thus exposing the right and left main bronchi. The subcarinal lymph node packet is dissected free with full visualization of the posterior membrane of the bronchus to avoid an injury. Next, the pleura anterior to the esophagus is incised, and the distal esophagus is completely mobilized at the level of the hiatus. A $1 / 2$ inch Penrose drain is then wrapped around the distal esophagus and used as a leash to manipulate the esophagus for circumferential exposure. The dissection is then carried cephalad towards the azygous vein, taking care to include the para-esophageal lymph nodes with the future specimen. The azygous vein is divided using the robotic stapler (gold tip load), and the dissection of the esophagus is continued toward the thoracic inlet. Ultimately achieving complete mobilization of the esophagus. The esophagus is then transected just below the level of the thoracic inlet sharply using the cutting tool on the robotic vessel sealer. Next, the gastric conduit is gently brought into the right thorax-taking care to avoid twisting during delivery (as a reference the staple line should be laterally positioned).

The gastric conduit is then divided away from the specimen using the robotic stapler (black load). A $1 \mathrm{~cm}$ gastrotomy is made $4 \mathrm{~cm}$ below the staple line of the 


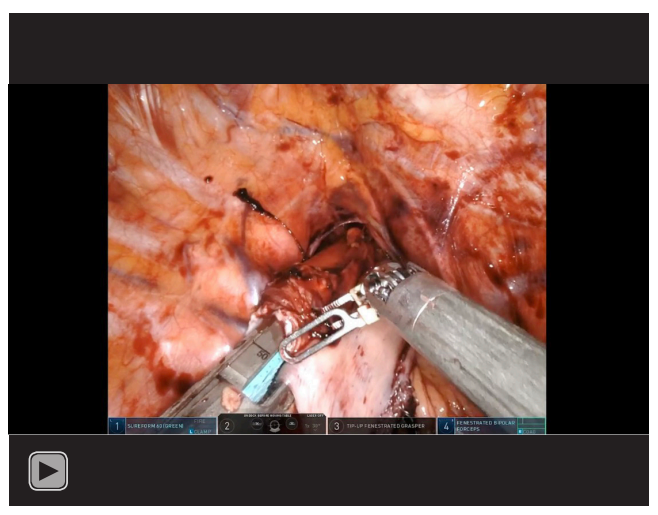

Video 4 Robotic Ivor Lewis esophagectomy (RILE) combined anastomosis.

conduit, and a side to side anastomosis is performed using the robotic stapler (typically using only $40 \mathrm{~mm}$ of the $60 \mathrm{~mm}$ load) - forming the posterior wall of the neo-esophagus as seen in Video 4. The anterior aspect is then closed in a hand-sewn fashion using an absorbable 2-0 V-Loc ${ }^{\mathrm{TM}}$ suture in two layers. During the outer layer closure, small bites of the omental flap are taken to buttress the anastomosis. At this point an EGD is routinely performed to assess the viability of the gastric conduit (neo-esophagus) and evaluate the patency of the anastomosis. Additionally, a leak test is performed by filling the right thorax with sterile water and insufflating the neo-esophagus endoscopically with carbon dioxide. Next, a straight $20 \mathrm{Fr}$ chest tube is placed posterior to the conduit towards the apex. The $19 \mathrm{Fr}$ Blake drain that was originally used as a buttress for the conduit delivery is then cut to appropriate length and placed alongside the conduit. In order to remove the esophagogastric specimen, the $15 \mathrm{~mm}$ assistant port is widened to a length of $5 \mathrm{~cm}$ with division of the underlying subcutaneous fat and muscle. An extra small Alexis ${ }^{\circledR}$ wound protector/retractor (Applied Medical, Rancho Santa Margarita CA, USA) is then placed and the specimen is removed with a ring forceps. Finally, a multilevel intrathoracic intercostal block (approximately $30 \mathrm{~mL}$ of $0.25 \%$ bupivacaine) is performed. The robot is then undocked and all incisions are closed. Of note, a nasogastric tube is not placed, as several studies have not demonstrated an increased risk of anastomotic leak or pulmonary complications with NGT omission (13).

\section{Postoperative care}

All patients are taken to the surgical intensive care unit for close monitoring postoperatively. Patients are placed on maintenance $\mathrm{pH}$ balanced intravenous fluids. For the first 24 hours, scheduled arterial blood gas and lactic acid samples are used to help dictate further resuscitation. Aiming to avoid hypovolemia given the consequence of malperfusion to the new esophagogastric anastomosis. Additionally, beginning postoperative day (POD) 2, a daily amylase level is collected from the Blake drain (placed along the length of the conduit) to assess for a leak. Patients are typically transferred to the general surgical floor on POD 4-5. At this time, the integrity of the anastomosis is tested by having the patient drink colored liquids and evaluating the quality of the chest tube and Blake drain output. A swallow study is not performed due to the high rate of false positives for an anastomotic leak (14). Once the patient has tolerated liquids for at least 24 hours, if there is no suspicion of a leak and output is minimal then both drains are removed. Patients are discharged home typically on POD 7 and remain on liquid diet. The post operative diet consists of 1 week of liquid diet, then soft diet for another 5 weeks before transitioning to a regular diet. Patients are routinely seen 1 week after discharge from the hospital, and again 1 week after starting a regular diet (therefore 7 weeks after discharge). Given the regimented dietary orders, a detailed educational diet packet is given to all esophagectomy patients preoperatively and an inpatient dietician will consult the patient towards the end of their hospitalization.

\section{Conclusions}

Since the initial conception of the esophagectomy, a variety of surgical approaches and anastomotic techniques have been developed. Often the anastomotic technique is dependent on the surgeon's comfort and experience. There is no clear consensus as to a superior technique, and disparity in the literature exists given the variations within each technique, the location of the anastomosis, and the use of induction therapy often implemented for esophageal cancer patients (15). Several randomized prospective trials comparing the hand-sewn technique to the stapled anastomosis (primarily circular stapled) have reported similar leak rates (15-17). Blackmon et al. compared handsewn, circular stapled, and modified Collard (also referred to as side-to-side stapled or combined stapled/sewn) anastomoses in a propensity-matched analysis, reporting no differences in leaks, perioperative morbidity, mortality, anastomotic recurrence, and overall survival rates (15). Ercan and colleagues compared the modified Collard 
technique to a hand-sewn anastomosis, demonstrating significantly lower rates of wound infection and reduced number of strictures requiring dilation with the modified Collard technique (8). Overall, a variety of techniques exist for esophageal surgery, and the operative technique continues to evolve. In our experience, the RILE with a combined sewn and stapled anastomosis is a safe and effective option for patients with mid to distal esophageal cancers.

\section{Acknowledgments}

Funding: None.

\section{Footnote}

Provenance and Peer Review: This article was commissioned by the Guest Editor (Douglas Z Liou) for the series "Advancement in Treatment for Esophageal Diseases" published in Fournal of Visualized Surgery. This article has undergone external peer review.

Conflicts of Interest: All authors have completed the ICMJE uniform disclosure form (available at https://jovs. amegroups.com/article/view/10.21037/jovs.2020.02.08/ coif). The series "Advancement in Treatment for Esophageal Diseases" was commissioned by the editorial office without any sponsorship or funding. The authors have no conflicts of interest to declare.

Ethical Statement: The authors are accountable for all aspects of the work in ensuring that questions related to the accuracy or integrity of any part of the work are appropriately investigated and resolved. All procedures performed in this study were in accordance with the Helsinki Declaration (as revised in 2013). The manuscript is waived from patient informed consent according to the ethics committee or institutional review board.

Open Access Statement: This is an Open Access article distributed in accordance with the Creative Commons Attribution-NonCommercial-NoDerivs 4.0 International License (CC BY-NC-ND 4.0), which permits the noncommercial replication and distribution of the article with the strict proviso that no changes or edits are made and the original work is properly cited (including links to both the formal publication through the relevant DOI and the license). See: https://creativecommons.org/licenses/by-nc-nd/4.0/.

\section{References}

1. Dubecz A, Schwartz SI. Franz John A. Torek. Ann Thorac Surg 2008;85:1497-9.

2. Straatman J, Van Der Wielen N, Cuesta MA, et al. Minimally Invasive Versus Open Esophageal Resection: Three-year Follow-up of the Previously Reported Randomized Controlled Trial the TIME Trial. Ann Surg 2017;266:232-6.

3. Yohannes P, Rotariu P, Pinto P, et al. Comparison of robotic versus laparoscopic skills: is there a difference in the learning curve? Urology 2002;60:39-45.

4. Cerfolio RJ, Bryant AS, Hawn MT. Technical aspects and early results of robotic esophagectomy with chest anastomosis. J Thorac Cardiovasc Surg 2013;145:90-6.

5. Ferrari-Light D, Geraci TC, Cerfolio RJ. Robotic Ivor Lewis esophagectomy: evolving technique to optimize outcomes. J Vis Surg 2019;5:41.

6. Price TN, Nichols FC, Harmsen WS, et al. A comprehensive review of anastomotic technique in 432 esophagectomies. Ann Thorac Surg 2013;95:1154-61.

7. Collard JM, Romagnoli R, Goncette L, et al. Terminalized semimechanical side-to-side suture technique for cervical esophagogastrostomy. Ann Thorac Surg 1998;65:814-7.

8. Ercan S, Rice TW, Murthy SC, et al. Does esophagogastric anastomotic technique influence the outcome of patients with esophageal cancer? J Thorac Cardiovasc Surg 2005;129:623-31.

9. Mallipeddi MK, Onaitis MW. The Contemporary Role of Minimally Invasive Esophagectomy in Esophageal Cancer. Curr Oncol Rep 2014;16:374.

10. van Workum F, van der Maas J, van den Wildenberg FJ, et al. Improved functional results after minimally invasive esophagectomy: intrathoracic versus cervical anastomosis. Ann Thorac Surg 2017;103:267-73.

11. Noussios G, Dimitriou I, Chatzis I, et al. The main anatomic variations of the hepatic artery and their importance in surgical practice: review of the literature. J Clin Med Res 2017;9:248.

12. Arya S, Markar S, Karthikesalingam A, et al. The impact of pyloric drainage on clinical outcome following esophagectomy: a systematic review. Dis Esophagus 2015;28:326-35.

13. Zhang R, Zhang L. Feasibility of complete nasogastric tube omission in esophagectomy patients. J Thorac Dis 2019;11:S819.

14. Tirnaksiz M, Deschamps C, Allen M, et al. Effectiveness of screening aqueous contrast swallow in detecting clinically 
significant anastomotic leaks after esophagectomy. Eur Surg Res 2005;37:123-8.

15. Blackmon SH, Correa AM, Wynn B, et al. Propensitymatched analysis of three techniques for intrathoracic esophagogastric anastomosis. Ann Thorac Surg 2007;83:1805-13.

16. Valverde A, Hay JM, Fingerhut A, et al. Manual versus

doi: 10.21037/jovs.2020.02.08

Cite this article as: Patel DC, Rodriguez E, Espinoza-Mercardo F, Imai TA, Soukiasian HJ. Robotic-assisted Ivor Lewis esophagectomy with combined stapled/sewn anastomosis. J Vis Surg 2020;6:46. mechanical esophagogastric anastomosis after resection for carcinoma: a controlled trial. Surgery 1996;120:476-83.

17. Law S, Fok M, Chu KM, et al. Comparison of hand-sewn and stapled esophagogastric anastomosis after esophageal resection for cancer: a prospective randomized controlled trial. Ann Surg 1997;226:169. 